\title{
Anfibios amenazados de Uruguay: efectividad de las áreas protegidas ante el cambio climático
}

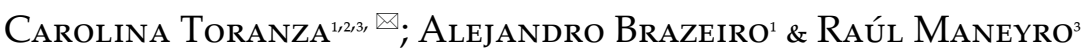 \\ ${ }^{1}$ Grupo Biodiversidad y Ecología de la Conservación, IECA, Facultad de Ciencias, Universidad de la República, Uruguay. CP: \\ 11600. ${ }^{2}$ Centro Interdisciplinario de Respuesta al Cambio y la Variabilidad Climática, Espacio Interdisciplinario, Universidad \\ de la República, Uruguay. ${ }^{3}$ Laboratorio de Sistemática e Historia Natural de Vertebrados, IECA, Facultad de Ciencias, \\ Universidad de la República, Uruguay
}

\begin{abstract}
Resumen. El cambio climático (CC) es una de las principales amenazas a la biodiversidad. Se han constatado efectos del CC sobre la distribución de las especies, principalmente corrimientos hacia latitudes altas y zonas elevadas. Las especies amenazadas resultan especialmente vulnerables a dichos cambios. En este marco, las áreas protegidas $(\mathrm{AP})$ podrían ser una herramienta clave para la adaptación al cambio climático. Nuestros objetivos son: estudiar los efectos del CC sobre la distribución y riqueza de anfibios amenazados y casi amenazados de Uruguay, y evaluar la eficacia del Sistema Nacional de Áreas Protegidas (SNAP) en el presente y ante escenarios de CC. Para modelar la distribución de nueve especies se obtuvieron registros de colecciones herpetológicas, publicaciones científicas y del GBIF, datos de clima actual y proyecciones del Modelo de Circulación General HadCM3 bajo los escenarios A2 y B2 del IPCC descargadas del Worldclim. La distribución potencial se obtuvo aplicando modelos de máxima entropía (MAXENT). Para evaluar la eficacia del SNAP se realizó un análisis de vacíos, superponiendo la cobertura de las AP con la distribución de las especies. Los modelos indican que la mayoría de las especies expandirá su distribución a futuro en Uruguay, excepto Pleurodema bibroni y Melanophryniscus montevidensis. La riqueza local de anfibios podría aumentar en el noroeste y este del país. Si bien los anfibios estudiados están incluidos al menos en un área del SNAP, la superficie protegida cubre menos de $2 \%$ de la distribución de las especies, tanto en la actualidad como bajo los escenarios de CC. Esto indica la baja eficacia del sistema. Si bien el CC esperado para la región no sería una amenaza para los anfibios estudiados, la escasa protección por parte del SNAP representa un riesgo para la conservación de la herpetofauna nativa frente a otros componentes del cambio global.
\end{abstract}

[Palabras clave: anuros, conservación, listas rojas, modelación de nicho]

Aвstract: Threatened amphibians of Uruguay: Effectiveness of protected areas face of climate change. Climate change (CC) constitutes one of the main biodiversity threats. The effects of CC on species are apparent worldwide, with primarily poleward and upward shifts in species distribution. Due to their narrow distribution, threatened species are highly vulnerable to CC. In this context, protected areas (PA) could be key tools for adaptation to CC. Our aims were to study the effects of CC on the distribution and richness of the threatened and near threatened amphibians of Uruguay; and to evaluate the effectiveness of the National Network of Protected Areas (NNPA) at present and under future climate scenarios. To model the distribution of nine species, we obtained records from herpetological collections, scientific publications and GBIF, as well as current data and future climate projections of the General Circulation Model HadCM3 under the A2 and B2 IPCC scenarios from Worldclim. To model species distribution we applied maximum entropy techniques (MAXENT). To evaluate the effectiveness of NNPA we conducted a gap analysis by overlaying PA with the distribution of species. The models indicate that the distribution of most amphibian species could expand in Uruguay in the future; except for Pleurodema bibroni y Melanophryniscus montevidensis. Local amphibian richness is predicted to increase, mainly in the northwest and east of the country. While amphibians studied are included in at least one PA, less than $2 \%$ of the distribution is actually covered by the NNPA, both currently and under CC scenarios; which it is a strong indicator of the inefficiency of the system. Although projected CC for this region would not constitute a major threat to amphibians studied, the scarce protection by the NNPA represents a risk to the conservation of herpetofauna facing the other components of global change.

[Keywords: anurans, conservation, red lists, niche modeling]

Editor asociado: Pedro Blendinger

ctoranza@gmail.com
Recibido: 18 de agosto de 2015

Aceptado: 16 de febrero de 2016 


\section{INTRODUCCIÓN}

El aumento de la concentración de gases de efecto invernadero en la atmósfera generó un incremento en la temperatura media global durante el siglo XX, que desencadenó una serie de alteraciones en el sistema climático (IPCC 2001). Numerosas evidencias indican efectos negativos del cambio climático a distintos niveles de organización biológica (Parmesan and Yohe 2003), desde subespecíficos (Balanyá et al. 2006) hasta modificaciones en ecosistemas terrestres y marinos (Boisvenue and Running 2006; Halpern et al. 2008). Se han constatado desplazamientos del rango de distribución en diversas especies animales y vegetales, principalmente en dirección a los polos (Parmesan 2006) y a zonas de mayor altitud (Pounds et al. 2006), que han generado extinciones locales e incluso globales (Pounds et al. 1999). Dado que se espera que el cambio en el clima varíe entre regiones y que las especies respondan de forma idiosincrática, resulta fundamental estudiar la distribución presente y proyectar los cambios a futuro para poder planificar $y$, eventualmente, mitigar los impactos sobre la biodiversidad (Araújo and Rahbek 2006). Este tipo de estudios son especialmente importantes en zonas de transición, donde por lo general coinciden los límites del rango de distribución de varias especies (Shmida and Wilson 1985). En dichas zonas es esperable que las dinámicas poblacionales estén más influenciadas por el clima que en el centro del rango de distribución (MacArthur 1972) y, por lo tanto, las poblaciones son más sensibles al cambio climático (Lovejoy and Hannah 2005).

Uruguay se ubica en el sudeste de Sudamérica en una zona de transición entre sistemas subtropicales y templados (Haretche et al. 2012), y constituye el borde de distribución para un importante número de anfibios (Maneyro and Kwet 2008). En esta zona se observaron aumentos en la temperatura y en las precipitaciones acumuladas anuales, así como de eventos extremos durante el siglo XX (Kane 2002; Rusticucci and Renom 2008; Marengo et al. 2010), y se espera que dichas tendencias se acentúen hacia finales de este siglo (DINAMA 2005). Uruguay constituye una región de transición entre biotas subtropicales y templadas; estudios previos indican que será una zona de alta tasa de recambio de anfibios (Lawler et al. 2009), por lo que es muy relevante evaluar los impactos potenciales del cambio climático sobre la distribución de la herpetofauna nativa.
Los cambios en la distribución son especialmente preocupantes en especies endémicas o amenazadas, que se caracterizan por su baja abundancia y/o distribución restringida (Gaston 1994). Según la Lista Roja de Especies Amenazadas de la Unión Internacional para la Conservación de la Naturaleza (UICN), los anfibios son actualmente el grupo de vertebrados que, en proporción, presenta más especies en categorías de amenaza global (UICN 2014). De un total de 6414 especies de anfibios evaluadas hasta 2014, 31\% están en alguna categoría de amenaza (i.e., vulnerable, amenazada o en peligro crítico), y $25 \%$ no cuenta con datos suficientes para su evaluación (UICN 2014). En lo que refiere a las tendencias, durante la evaluación del Global Amphibian Assessment en 2004 se constató que de las 5743 especies evaluadas, 1430 especies (25\%) sufrieron disminuciones poblacionales respecto a evaluaciones previas. Por otra parte, se observó que 435 especies que declinaron de forma rápida aumentaron su categoría de amenaza respecto a la década del 80, y casi la mitad de ellas lo hicieron por causas no identificadas, presuntamente vinculadas al cambio climático y a enfermedades (Stuart et al. 2004). Los factores causales de la declinación de las poblaciones de anfibios son múltiples; la modificación y pérdida de hábitat es la causa principal (Todd et al. 2009). El cambio climático se encuentra entre las causas adicionales de este fenómeno, y se ha indicado su sinergia con otras amenazas tales como la expansión de la quitridiomicosis (Bosch et al. 2007).

Las áreas protegidas son una de las herramientas principales de conservación a nivel mundial (Gaston et al. 2008) y, en general, se establecen en sitios de alta riqueza y endemicidad de especies (Myers et al. 2000). Este criterio de selección ha sido recientemente criticado dado que los sitios de alta concentración de especies no necesariamente coinciden con la distribución de las especies amenazadas (Deguise and Kerr 2005; Villalobos et al. 2013). La ubicación actual de las áreas protegidas asume que las distribuciones de las especies cambian de forma relativamente lenta. Sin embargo, se está tomando conciencia que la selección de sitios de conservación debe considerar el cambio climático y evaluar posibles modificaciones respecto a la distribución actual de las especies (Araújo and Rahbek 2006). Nuestro trabajo tiene dos objetivos 
principales: 1) analizar los efectos potenciales de los cambios climáticos proyectados para Uruguay sobre la distribución de anfibios autóctonos amenazados; 2) evaluar la efectividad del Sistema Nacional de Áreas Protegidas de Uruguay (SNAP) para proteger a los anfibios amenazados, en el presente y en escenarios futuros de cambio climático.

\section{MATERIALES y MÉTODOS}

\section{Área de estudio}

Uruguay se ubica al SE de Sudamérica $\left(30^{\circ}\right.$ $\left.35^{\circ} \mathrm{S}, 53^{\circ}-58^{\circ} \mathrm{W}\right)$. Su superficie terrestre es de $176215 \mathrm{~km}^{2}$. La topografía está dominada por colinas suaves y por una densa red hidrográfica (Achkar and Domínguez 2000). Desde el punto de vista biogeográfico, Uruguay se ubica en la Provincia Pampeana y recibe influencias de las provincias Chaqueña y Paranaense (Maneyro and Kwet 2008; Haretche et al. 2012). Según la clasificación climática Köppen, Uruguay se incluye en la categoría "Cfa", con un clima templado y húmedo con veranos calientes (Kottek et al. 2006). La temperatura media anual es $17.5^{\circ} \mathrm{C}$ y las precipitaciones medias, que se distribuyen uniformemente a lo largo del año, varían entre 1100 y 1400 mm/año (DINAMA 2005).

\section{Bases de datos biológicos}

Inicialmente elaboramos una lista de anuros amenazados y casi amenazados a nivel global y / o nacional. A nivel global nos basamos en la Lista Roja de Anfibios de la UICN (UICN 2014), y a nivel nacional tomamos como referencia la Lista Rojas de Anfibios y Reptiles de Uruguay (Carreira and Maneyro 2015). Luego elaboramos una base de datos con información de registros históricos (posteriores a 1940) de las principales colecciones herpetológicas del país: 1) Colección de Zoología de Vertebrados de Facultad de Ciencias (CZVB), y 2) Colección del Museo de Historia Natural (MNHN). Los registros del MNHN se obtuvieron a partir del trabajo de Núñez et al. (2004). Como la mayoría de las especies no son endémicas de Uruguay, se obtuvieron registros de las especies en el Neotrópico en el portal Global Biodiversity Information Facility (GBIF, www.gbif.org), que facilita el acceso a registros de colecciones científicas mundiales. Adicionalmente, se utilizaron publicaciones científicas que reportaran la localización de registros de las especies (Rosset 2008; Mendonça do Prado et al. 2009;
Zank et al. 2013). Dado que muchas de las especies amenazadas cuentan con registros escasos, no es posible modelar su distribución. En este trabajo modelamos la distribución potencial de especies que cuentan con al menos cinco registros, por lo que modelamos la distribución de nueve de los 17 anfibios amenazados de Uruguay (Tabla 1 y 2).

\section{Información ambiental}

Para modelar la distribución actual y futura de las especies utilizamos 36 variables climáticas (temperaturas máximas y mínimas mensuales, y precipitación mensual) y la altitud, con una resolución espacial de 2.5 minutos $(\sim 5 \mathrm{~km})$. La información se obtuvo de la base de datos climáticos Worldclim (Hijmans et al. 2005; www.worldclim.org). La información actual corresponde a modelaciones basadas en lo observado en el período 1950-2000, y la futura a la salida del modelo de circulación global HadCM3 del Hadley Centre para los escenarios A2 (severo) y B2 (moderado) del IPCC, horizontes temporales 2050 y 2080. Existen múltiples incertidumbres asociadas al modelado de distribuciones futuras vinculadas a la elección de los escenarios socioeconómicos futuros y del o los Modelos de Circulación General (MCG), además de las incertidumbres inherentes al sistema climático (Mitchell et al. 1999). En nuestra región se han hecho varios estudios evaluando la adecuación de distintos MCG al clima de la región (MGAP-FAO 2013). En este trabajo seleccionamos la salida del modelo HadCM3 porque los estudios climatológicos regionales indican que es el que mejor reproduce la climatología observada respecto a las variables temperatura y precipitación (DINAMA 2005; Bidegain and Camilloni 2006). Asimismo, el HadCM3 es el MCG utilizado actualmente por el Sistema Nacional de Respuesta al Cambio Climático de Uruguay (SNRCC) en los estudios de adaptación al cambio climático (MGAP-FAO 2013).

\section{Modelación de las distribuciones}

Los datos de distribución de especies suelen ser incompletos, y en general sólo se cuenta con información de presencia. Salvo en especies comunes o extensamente estudiadas, el número de registros por especie suele ser bajo (Pearson et al. 2007). Una de las herramientas más difundidas en la actualidad para la estimación de distribuciones potenciales son los Modelos de Envoltura 
Tabla 1. Lista de especies de anuros amenazados y casi amenazados de Uruguay. Se indica la categoría de amenaza global (UICN) y nacional (Carreira and Maneyro 2015). Para cada especie se indica: Familia, nombre común y la categoría de amenaza global y nacional según criterios de la UICN (PM=preocupación menor; DI=datos insuficientes, $\mathrm{CA}=$ casi amenazada, $\mathrm{VU}=$ vulnerable, $\mathrm{A}=$ amenazada, $\mathrm{PC}=$ peligro crítico, $\mathrm{NE}=$ no evaluada).

* Especies modeladas en este estudio (con cinco o más localidades).

Table 1. List of threatened and near threatened anuran species in Uruguay. For each species taxonomic family, common name, and its global and national threat category according to IUCN criteria (PM=least concern; DI=data deficient, $\mathrm{CA}=$ near threatened, $\mathrm{VU}=$ vulnerable, $\mathrm{A}=$ threatened, $\mathrm{PC}=$ critical risk and $\mathrm{NE}=$ not evaluated) are indicated.

* Species modeled in this study (present in at least five localities).

\begin{tabular}{|c|c|c|c|c|}
\hline \multirow[t]{2}{*}{ Familia } & \multirow[t]{2}{*}{ Especie } & \multirow[t]{2}{*}{ Nombre común } & \multicolumn{2}{|c|}{ Grado de amenaza } \\
\hline & & & Global & Nacional \\
\hline \multirow[t]{6}{*}{ Hylidae } & \multicolumn{2}{|c|}{$\begin{array}{l}\text { Argenteohyla siemersi (Mertens,Rana motor } \\
\text { 1937) }\end{array}$} & A & DI \\
\hline & \multicolumn{2}{|c|}{$\begin{array}{l}\text { Dendropsophus minutus(Peters, Ranita rayada } \\
\text { 1872)* }\end{array}$} & PM & A \\
\hline & $\begin{array}{l}\text { Dendropsophus nanus } \\
\text { (Boulenger, } 1889)^{*}\end{array}$ & Ranita enana del Chaco & PM & A \\
\hline & Lysapsus limellum Cope, $1862^{*}$ & Rana boyadora chica & PM & A \\
\hline & $\begin{array}{l}\text { Scinax aromothyella Faivovich, } \\
2005\end{array}$ & Ranita de las tormentas & DI & A \\
\hline & Scinax nasicus (Cope, 1862)* & Ranita de pecho manchado & PM & A \\
\hline \multirow[t]{6}{*}{ Bufonidae } & $\begin{array}{l}\text { Melanophryniscus devicenzii } \\
\text { Klappenbach, } 1968\end{array}$ & Sapito de Rivera & A & A \\
\hline & $\begin{array}{l}\text { Melanophryniscus langonei } \\
\text { Maneyro, Naya \& Baldo, } 2008\end{array}$ & Sapito de Langone & PC & PC \\
\hline & $\begin{array}{l}\text { Melanophryniscusmontevidensis } \\
\text { (Philippi, 1902)* }^{*}\end{array}$ & Sapito de Darwin & VU & PC \\
\hline & \multicolumn{2}{|c|}{$\begin{array}{l}\text { Melanophryniscus pachyrhynusSapito de nariz gorda } \\
\text { (Miranda-Ribeiro, 1920) }\end{array}$} & DI & A \\
\hline & $\begin{array}{l}\text { Melanophryniscus sanmartini } \\
\text { Klappenbach, } 1968^{*}\end{array}$ & Sapito de San Martín & CA & CA \\
\hline & $\begin{array}{l}\text { Rhinella achavali (Maneyro, } \\
\text { Arrieta \& de Sá, 2004)* }\end{array}$ & Sapito de Achaval & PM & CA \\
\hline Ceratophryidae & \multicolumn{2}{|c|}{ Ceratophrys ornata (Bell, 1843) Escuerzo } & CA & A \\
\hline Cycloramphidae & \multicolumn{2}{|c|}{$\begin{array}{l}\text { OdontophrynusmaisumaRosset,Escuercito } \\
2008\end{array}$} & NE & $\mathrm{CA}$ \\
\hline \multirow[t]{3}{*}{ Leptodactylidae } & \multicolumn{2}{|c|}{$\begin{array}{l}\text { Leptodactylus furnarius (SazimaRana de Campo grande } \\
\text { y Bokerman, 1978)* }\end{array}$} & PM & PC \\
\hline & $\begin{array}{l}\text { Physalaemus fernandezae } \\
\text { (Muller, 1926) }\end{array}$ & Ranita de Fernández & PM & A \\
\hline & $\begin{array}{l}\text { Pleurodema bibroni (Tschudi, } \\
1838)^{*}\end{array}$ & Ranita de Bibron & CA & CA \\
\hline
\end{tabular}

Climática (Lobo et al. 2010). Éstos estiman la distribución potencial correlacionando información de ocurrencia de las especies con predictores ambientales. Estudios recientes, enfocados en la comparación de distintos métodos de modelado, indican que el programa MAXENT, que aplica una técnica de aprendizaje automático y utiliza un algoritmo de máxima entropía (Phillips et al. 2004), es uno de los métodos más robustos (Elith et al. 2006; 2011).

En este trabajo utilizamos la versión 3.3.1 de Maxent para modelar la distribución de los nueve anfibios amenazados (www.cs.princeton.edu/ schapire/maxent).
Inicialmente, los modelos se corrieron utilizando registros de todo el rango de distribución conocido de las especies. Sin embargo, debido a los grandes errores de comisión de los modelos, en especial en el límite sur de distribución, se decidió modelar la distribución presente y futura de las especies en base a registros provenientes de la región climática $\mathrm{Cfa}$, que incluye a Uruguay, sur de Brasil y Paraguay y centro de Argentina (Kottek et al. 2006, Información Suplementaria). El programa se corrió 100 veces utilizando las características por defecto: 500 iteraciones, eliminación de registros duplicados, umbral de convergencia $=0.00001$ 
y el parámetro de regularización $\beta=1$ (Phillips et al. 2006). Para cada especie se eligió el modelo con mejor grado de predicción (AUC). En lo que refiere a la interacción entre las variables ambientales, se seleccionaron: relaciones lineales, cuadráticas y productos (Phillips and Dudik 2008). Para validar los modelos los datos de registros se dividieron en una muestra de entrenamiento (70\%) y una de prueba (30\%), excepto en el caso de Lysapsus limellum, que se modeló con menos de 10 registros (Tabla 1). La precisión del modelo la evaluamos mediante el "Área Bajo la Curva" (AUC por su sigla en inglés), derivada de la curva de características operativas (o curva ROC), que mide el compromiso entre la sensibilidad y la especificidad del modelo (Phillips and Dudik 2008). Los modelos son considerados buenos cuanto más próximo se encuentra su AUC a 1 (Phillips et al. 2004).

Como resultado del proceso de modelación se obtuvo la distribución potencial de cada especie para la región. Las distribuciones de las especies fueron convertidas a un mapa de presencia/ausencia utilizando inicialmente el umbral de corte Minimum Training Presence, sugerido para especies con bajo número de localidades (Hernández et al. 2006; GomesCortés 2009). Comolos modelos dedistribución de especies se basan principalmente en datos de presencias (Franklin 2010), para obtener distribuciones más cercanas a la distribución realizada y disminuir los errores de omisión y de comisión aplicamos la metodología propuesta por Pineda and Lobo (2009), que se basa en la selección de celdas bien muestreadas que permitan identificar "ausencias verdaderas". Inicialmente, pre- seleccionamos aquellas celdas del Plan Cartográfico Nacional que cuentan con 100 ó más registros de anfibios, lo cual ha sido indicado como un buen esfuerzo de muestreo en el grupo (Canavero et al. 2010a). Luego, para cada celda se construyó una matriz de registros por especie y se aplicaron distintos estimadores de riqueza (Chao1, Chao2 y Jackknife) usando el programa EstimateS v8.2 (ver Información Suplementaria). Las celdas con una riqueza observada igual o mayor al 75\% de la riqueza máxima estimada fueron consideradas como "celdas bien muestreadas", y en ellas, las ausencias de registro de una especie se consideraron como verdaderas (Información Suplementaria). Si el modelo indica la presencia de una especie en una celda bien muestreada donde está ausente, se aumenta el umbral de corte del modelo para excluir esa celda y disminuir la sobreestimación en la distribución. Las distribuciones modeladas fueron cruzadas con una capa correspondiente al límite terrestre de Uruguay. Posteriormente, la variación espacial del número de anfibios amenazados se mapeó a escala 1:50000 utilizando la grilla del Plan Cartográfico Nacional de Uruguay.

Para evaluar la coincidencia espacial de las áreas protegidas del SNAP con la distribución de anfibios amenazados, en la actualidad y ante escenarios de cambio climático, se descargaron las coberturas con los límites de las áreas protegidas de la página del Ministerio de Vivienda, Ordenamiento Territorial y Medio Ambiente (www.mvotma.gub.uy), (Figura 1). Luego se realizó un análisis de vacíos (Scott and Schipper 2006) superponiendo las distribuciones potenciales de las especies,

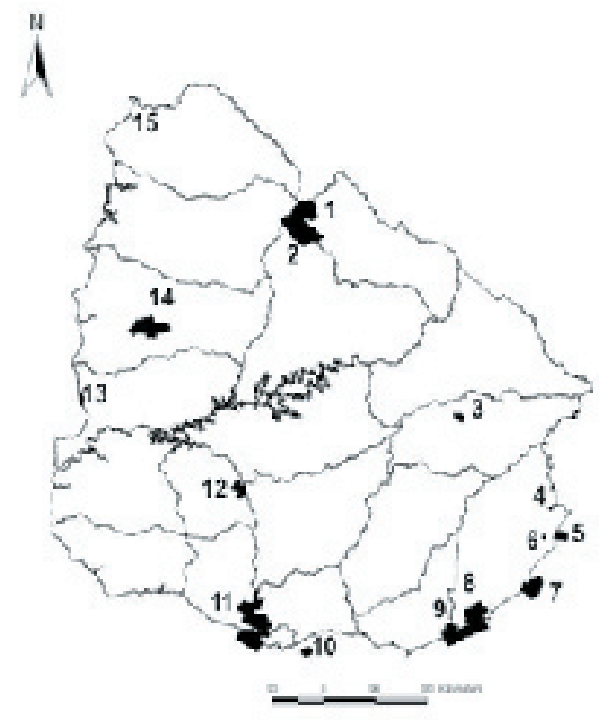

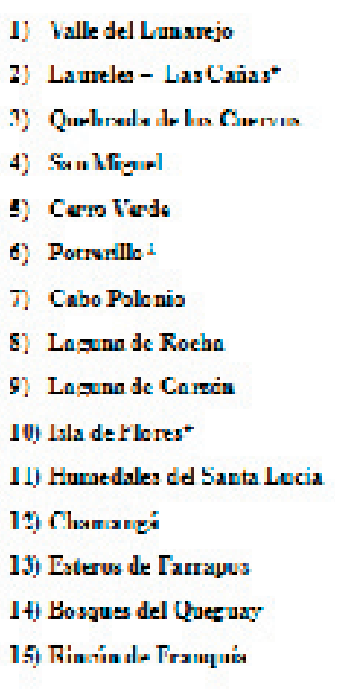

alle del Lansrej

1) Oumbretle le bo Curvo

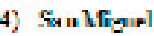

Cerro Verde

Portwrillo

Loguna de Roeha

Loguna de Corrci

10) Lsla de Flores"

1010 Hurotales ded Lanta lecia

12) Clusanaz:

13) Eaterus de Tamrapus

15) Rincínal- Trampuí
Figura 1. Distribución espacial de las áreas protegidas del Sistema Nacional de Áreas Protegidas (SNAP) de Uruguay.

* Áreas que se encuentran en proceso de ingreso al SNAP.

Figure 1. Spatial distribution of the protected areas within the National System of Protected Areas (SNAP) of Uruguay.

*Areas in the process of admission to SNAP 
actuales y futuras bajo escenarios de CC, con la cobertura espacial actual de SNAP de Uruguay, en un entorno de Sistema de Información Geográfica.

\section{Resultados}

\section{Efectos del CC sobre la distribución de anfibios amenazados}

Los modelos de distribución presentaron muy buenos ajustes en todos los casos, con valores de AUC mayores a 0.95 (Tabla 2). Las variables que más pesaron en los modelos fueron la altitud, la precipitación en meses cálidos y la temperatura mínima en meses fríos (Tabla 2). El análisis en base a las celdas bien muestreadas indicó una sobreestimación de la distribución de Melanophyiniscussanmartiniy Dendropsophus minutus, por lo que en ambos casos se aumentó el umbral de corte. Las distribuciones modeladas coincidieron con propuestas de distribución previas generadas a partir de la opinión de expertos (UICN 2014) o de modelos (Bernardo-Silva et al. 2012; Zank et al. 2014). Los mapas de distribución actual y futura de cada especie se presentan en la Información Suplementaria.

El número actual de especies amenazadas varió en el espacio entre 0 y 6 especies (Figura 2A). Las zonas con mayor número de anfibios amenazados se localizaron en: 1) Noroeste, en la planicie de inundación del Río Uruguay y su confluencia con el Río Cuareim, 2) Este, en serranías y quebradas, y 3) Sureste, en la costa atlántica y al sistema de lagunas y humedales costeros (Figura 2A).
Los resultados de la modelación indicaron que bajo escenarios de clima futuros, la mayoría de las especies expandirían su rango de distribución en Uruguay hacia mediados del siglo XXI (Tabla 2). Asimismo, se espera que hacia mediados de siglo ocurra un aumento de la riqueza local de los anfibios actualmente amenazados (Figura 2). Las proyecciones de cambio son similares en los dos escenarios, aunque más marcadas en el escenario A2 (Figura 2). Sin embargo, ambos escenarios (A2 y B2) proyectan una disminución en el rango de distribución de Melanophryniscusmontevidensisy Pleurodema bibroni(Tabla2).EnelcasodeM.montevidensis, las proyecciones indican la pérdida total de las condiciones climáticas que ocupa actualmente (Figura S5).

\section{Efectividad presente y futura del SNAP}

En la actualidad, el SNAP ocupa $1804 \mathrm{~km}^{2}$, lo que representa $0.57 \%$ de la superficie de Uruguay (MVOTMA/SNAP 2015). De acuerdo al análisis de vacíos, todas las especies modeladas tendrían cubierta su distribución actual con al menos un área protegida (Figura 3). Las especies con menor cobertura son Dendropsophus nanus y Scinax nasicus (con un área) y Dendropsophus minutus, Lysapsus limellum y Leptodactylus furnarius (con tres áreas protegidas cada una). La proporción del área de distribución de estas especies incluida en áreas protegidas es menor a $1 \%$. Los porcentajes de distribución protegida no mejoran de manera sustancial en el caso de las especies mejor representadas en el SNAP, donde la cobertura fue inferior a $2 \%$.

Tabla 2. Resultados de la modelación con Maxent de la distribución de nueve especies de anuros amenazados indicando el número de localidades utilizado, el valor del Área bajo la Curva (AUC) y la tendencia esperada en la distribución según las proyecciones de cambio climático (escenarios socioeconómicos A2 y B2) resultados de la modelación futura. * La tendencia de los cambios proyectados fue la misma en ambos escenarios (A2 y B2) del IPCC, con excepción de Dendropsophus minutus.

Table 2. Modeling results (Maxent) of the distribution of threatened species, indicating: the number of localities, the area under the curve (AUC) and the trend of distribution under climate change (A2 and B2 socioeconomic scenarios).

* The trend of projected changes was the same in both studied scenarios (A2 and B2) of the IPCC, with the exception of Dendropsophus minutus

\begin{tabular}{lccc}
\hline \multicolumn{1}{c}{ Especie } & № localidades & AUC & Cambios proyectados* $^{*}$ \\
\hline Dendropsophus minutus & 27 & 0.975 & Retracción/Expansión \\
Dendropsophus nanus & 26 & 0.969 & Expansión \\
Lysapsus limellum & 8 & 0.993 & Expansión \\
Scinax nasicus & 33 & 0.972 & Expansión \\
Melanophryniscus montevidensis & 35 & 0.998 & Retracción \\
Melanophryniscus sanmartini & 13 & 0.999 & Expansión \\
Rhinella achavali & 19 & 0.992 & Expansión \\
Leptodactylus furnarius & 13 & 0.973 & Expansión \\
Pleurodema bibroni & 29 & 0.998 & Retracción \\
\hline
\end{tabular}




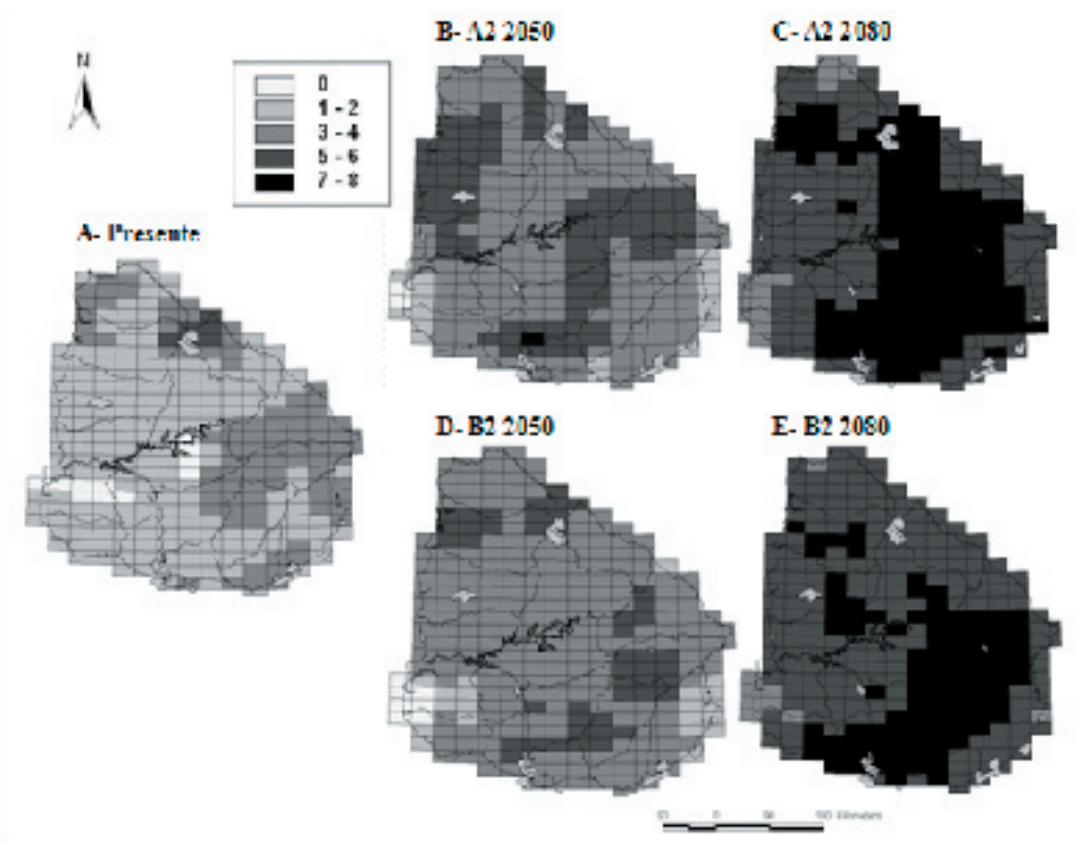

Figura 2. Riqueza potencial de anfibios amenazados en el presente y según las proyecciones climáticas futuras bajo los escenarios A2 y B2 del IPCC. Se muestra la ubicación de las Áreas Protegidas en Uruguay.

Figure 2. Potential species richness of threatened amphibians at the present and under climatic projections of two scenarios (A2 and B2) of the IPCC for 2050 and 2080. Protected Areas are shown as hatched polygons.

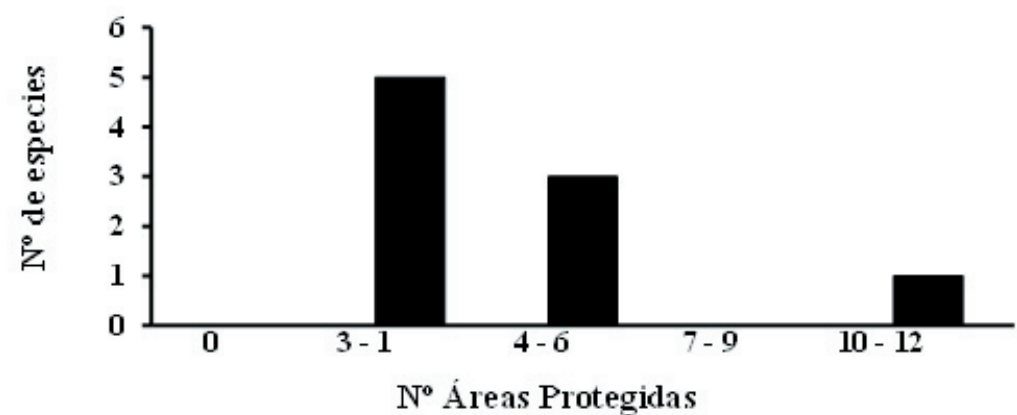

Figura 3. Grado de cobertura de los anfibios amenazados por el Sistema Nacional de Âreas Protegidas (SNAP) de Uruguay. Se muestra información para las nueve especies modeladas.

Figure 3. The coverage of threatened amphibians within the National System of Protected Areas (SNAP) in Uruguay for the nine modeled species.
Debido a que se espera que la mayoría de las especies expandan su área de distribución, el grado de cobertura por parte del SNAP se mantendría en el futuro o incluso aumentaría en los escenarios evaluados, a excepción de M. montevidensis.

\section{DISCUSIÓN}

\section{Efectos potenciales del CC}

Durante el siglo XX ocurrieron corrimientos en los rangos de distribución de las especies a causa del cambio climático, y se prevé que dichos cambios se acentúen durante este siglo (Parmesan 2006). Nuestros resultados indican que la mayoría de los anfibios estudiados podrían expandir su distribución en Uruguay en función de las proyecciones climáticas del IPCC para mediados y finales del siglo XXI. Estudios anteriores sobre proyecciones de cambio climático indican que la mayoría de los anfibios neotropicales sufrirá contracciones en su distribución, principalmente en las zonas tropicales y subtropicales del continente (Lawler et al. 2009; Blaustein et al. 2010). A pesar de la incongruencia aparente entre estas predicciones, se debe tener en cuenta que Uruguay representa el límite sur del rango de distribución de muchas especies $y$, por ende, se encuentra en la zona para la que se proyectan ganancias de especies bajo distintos escenarios del IPCC (Lawler et al. 2009). Los corrimientos de la distribución de especies hacia latitudes más altas han sido documentados en múltiples taxones, 
en especial en el hemisferio norte (Parmesan 2006; Chen et al. 2011). Dada la ubicación de Uruguay, el cambio climático podría generar una ventana de oportunidad para la expansión geográfica de los anfibios amenazados, lo cual podría repercutir favorablemente en su estatus de conservación a nivel nacional.

La probabilidad de que efectivamente se den expansiones de las especies a futuro dependerá de varios factores, y resultará clavela capacidad de dispersión de los individuos. En este sentido, se han formulado distintas hipótesis de dispersión de las especies (Peterson et al. 2001). Algunos autores plantean que en el caso de taxones con escasa capacidad de dispersión, como los anfibios, y ante el aumento de la fragmentación de hábitats, lo más adecuado es considerar la hipótesis de no-dispersión de las especies (Araújo et al. 2006; D'Amen et al. 2011). Nuestros resultados indican que incluso considerando la no-dispersión, la mayoría de las especies estudiadas no disminuiría su distribución actual en Uruguay bajo los escenarios de cambio climático evaluados. Como consecuencia de los cambios previstos en su distribución, también se espera un aumento local del número de especies en los dos escenarios climáticos estudiados (A2 y B2). Esto último coincide con los proyectado para la riqueza de anfibios en esta región (Blaustein et al. 2010; Toranza et al. 2012).

La principal explicación a los pronósticos de expansión en la distribución de los anfibios radica en su estrecho vínculo con el clima. Los anfibios son muy sensibles a las condiciones ambientales y están limitados principalmente por la temperatura y la humedad (Carey and Alexander 2003). Para la mayoría de los anfibios en este estudio, la precipitación de los meses cálidos y la temperatura mínima de los meses fríos fueron las variables de mayor peso en los modelos. Esto sugiere que la distribución de las especies está limitada por dichas variables o variables asociadas a temperatura y precipitación (e.g., humedad, evapotranspiración). Por lo tanto, los aumentos en la precipitación y en la temperatura proyectados para Uruguay generarían condiciones favorables para su expansión geográfica. Además de la expansión de las especies presentes en Uruguay, una "tropicalización del clima" podría favorecer la dispersión hacia el sur de especies de climas tropicales y subtropicales. En particular, podría representar una oportunidad para especies distribuidas en el sur de Brasil, perteneciente a la fauna riograndense y del bosque atlántico (Maneyro and Kwet 2008).
Aunque a priori el cambio climático no tendría impactos negativos para la mayoría de las especies en Uruguay, P. bibroni y $M$. montevidensis sufrirían una retracción en su distribución. Si se considera el corrimiento hacia el sur de las condiciones climáticas adecuadas para estas especies y que el Río de la Plata y el océano Atlántico constituyen una barrera geográfica, los cambios proyectados implican un estrechamiento de sus rangos de distribución. En el caso de M. montevidensis, el corrimiento hacia el sur implicaría la pérdida total de las condiciones climáticas favorables para la especie (Toranza and Maneyro 2013; Zank et al. 2014). P. bibroni se distribuye principalmente en el sur de Uruguay, pero ha sido reportada para el sur de Brasil y hay un registro histórico en el noreste de Paraguay (Kolenc et al. 2012). En los últimos años la especie ha sufrido un importante declive a causa del fuerte impacto antrópico en la zona costera (Silvano et al. 2004). También se ha señalado su desaparición en hábitats relativamente inalterados (Kolenc et al. 2009), y se plantean como posibles causas la quitridiomicosis y el cambio climático (Kolenc et al. 2012). Bardier et al. (2011) reportaron la presencia del hongo Batrachochytrium dendrobatidis en especímenes adultos de esta especie en Cerro Verde, una de las áreas protegidas costero-marinas de Uruguay. Este hallazgo representa una gran preocupación sobre la condición de las poblaciones de $P$. bibroni en ambientes naturales. Nuestros resultados apoyan el planteo de que el cambio climático es una amenaza para esta especie, ya que, según nuestros modelos, restringirá su distribución a mediano plazo. Por lo tanto, constituyen un nuevo elemento para que se revise el estatus global de conservación de $P$. bibroni y se tomen medidas concretas que aseguren su conservación a futuro (Natale and Maneyro 2008; Bardier et al. 2011; Kolenc et al. 2012).

M. montevidensis es exclusiva de ambientes psamófilos de la costa atlántica de Uruguay y sur de Brasil, que han sido muy modificados por la urbanización y la forestación. Actualmente, está categorizada a nivel mundial como vulnerable, y en peligro crítico en Uruguay (Carreira and Maneyro 2015). Nuestros modelos prevén una desaparición total de las condiciones climáticas donde se distribuye $M$. montevidensis, lo que podría provocar su extinción hacia mediados de siglo. Debido a su reproducción explosiva en charcos temporales la especie es muy 
dependiente de la temperatura y precipitación en los meses cálidos, lo que ha sido planteado como el mayor riesgo frente a las previsiones de cambio climático en la región (Toranza and Maneyro 2013). Si bien la especie está protegida por varias AP (Figura S2), se ha planteado la necesidad de incluir nuevas áreas para asegurar una mayor representatividad de las distintas poblaciones y diversidad genética de la especie (Bernardo-Silva et al. 2012). Dadas las previsiones de los modelos, es fundamental aumentar nuestro conocimiento respecto a la fisiología y autoecología de esta especie para establecer un manejo efectivo (Toranza and Maneyro 2013; Zank et al. 2014).

\section{Efectividad del SNAP para proteger a los anfibios amenazados}

Uno de los objetivos fundamentales de la planificación sistemática para la conservación es la protección de una muestra representativa del ensamble de especies de una región determinada (Margules and Pressey 2000). El SNAP de Uruguay tiene dentro de sus objetivos proteger la diversidad biológica, priorizando la conservación de las poblaciones de flora y fauna autóctonas en peligro o amenazadas de extinción (www.mvotma.gub.uy/snap). Nuestro estudio indica que todos los anfibios amenazados están incluidos en algún área del SNAP, como fue corroborado en los inventarios faunísticos recientes (www.snap.gub.uy/ especies). Sin embargo, la escasa área de distribución de las especies bajo protección del SNAP $(<2 \%)$ indica una baja eficacia del sistema. Esto está estrechamente ligado a la baja superficie protegida en Uruguay, que es el país con menor porcentaje de cobertura por áreas protegidas de América Latina (Elbers 2011).

En el contexto actual de transformación antrópica del paisaje, resulta preocupante la baja superficie destinada en Uruguay a la conservación o manejo de especies amenazadas. Varios trabajos señalan que el cambio en el uso del suelo es la principal amenaza para los anfibios en Uruguay (Maneyro and Langone 2001; Langone et al. 2006; Canavero et al. 2010b). A modo de ejemplo, un estudio reciente del efecto del uso del suelo actual y bajo un escenario de cambio de uso del suelo para las próximas décadas, indica que se espera un aumento importante de la superficie de cultivos agrícolas y forestales en el área de distribución conocida de Melanophryniscus sanmartini (Rosenstock et al. 2015), una de las especies aquí estudiadas.

El presente estudio aporta información sobre el grado de protección de los anfibios amenazados en Uruguay y su situación frente a las proyecciones de cambio climático en ésta región. Según nuestros resultados el cambio climático no representaría una amenaza generalizada para las poblaciones de anfibios que viven en Uruguay, al menos bajo los escenarios evaluados y según las proyecciones de MCG HadCM3. Sin embargo, nuestros resultados señalan que Melanophryniscus montevidensis y Pleurodema bibroni podrían sufrir retracciones en su distribución, por lo que es necesario desarrollar medidas de manejo activas que aseguren su conservación a largo plazo. El SNAP protege una mínima área de la distribución de los anfibios amenazados, lo que es un fuerte indicador de la ineficacia del sistema y representa un llamado de atención frente al acelerado cambio en el uso del suelo y la expansión de cultivos agrícola forestales sobre los hábitats naturales de éstas especies.

Agradecimientos. Carolina Toranza agradece a la OEA y a la ONG Fondo Verde por el otorgamiento de la Beca OAS/DHDEC/ CIR.233/.

\section{ReFERENCIAS}

Achkar, M., and A. Domínguez. 2000. El sistema ambiental uruguayo. Pp. 17-27 en A. Domínguez y R. Prieto (eds.). Perfil ambiental del Uruguay. Vol. 1. 1a ed. Editorial Nordan-Comunidad. Montevideo, Uruguay. Pp. 269.

Alford, R., and S. J. Richards. 1999. Global amphibian declines: a problem in applied ecology. Annu Rev Ecol Evol Syst 30:133-165.

Araújo, M., and C. Rahbek. 2006. How does climate change affect biodiversity? Science 313:1396-1397.

Araújo, M., W. Thuiller, and R. Pearson. 2006. Climate warming and the decline of amphibians and reptiles in Europe. J Biogeogr 33:1712-1728.

Balanyá, J., J. Oller, R. Huey, G. Gilchrist, and L. Serra. 2006. Global genetic change tracks global climate warming in Drosophila subobscura. Science 313:1973-1975.

Bardier, C., R. Ghirardi, M. Levy, and R. Maneyro. 2011. First case of chytridiomycosis in an adult specimen of a native anuran from Uruguay. Herpetol Rev 42:65-66.

Bernardo-Silva, J., C. Martins-Ferreira, R. Maneyro, and T. Ochotorena de Freitas. 2012. Identification of priority areas 
for conservation of two endangered parapatric species of Red-bellied toads using ecological niche models and hotspot analysis. Nat Conservação 10:207-213.

Bidegain, M., and I. Camilloni. 2006. Performance of GCMs and climate future scenarios for Southeastern South America. Pp. 223-226 en International Conference on Southern Hemisphere Meteorology and Oceanography (ICSHMO), 8, Foz do Iguaçu. Proceeding. Sao José dos Campos: IMPE.

Blaustein, A., S. Walls, B. Bancroft, J. Lawler, C. Searle, and S. Gervasi. 2010. Direct and indirect effects of climate change on amphibian populations. Diversity 2:281-313.

Boisvenue, C., and S. Running. 2006. Impacts of climate change on natural forest productivity evidence since the middle of the 20th century. Glob Chang Biol 12:862-882.

Bosch, J., L. Carrascal, L. Durán, S. Walker, and M. Fisher. 2007. Climate change and outbreaks of amphibian chytridiomycosis in a montane area of Central Spain; is there a link? Proc R Soc Lond B Biol Sci 274:253-260.

Brazeiro, A., M. Achkar, C. Toranza, and L. Barthesagui. 2008. Potenciales impactos del cambio de uso de suelo sobre la biodiversidad terrestre de Uruguay. Pp. 7-22 en A. Volpedo y L. Fernández-Reyes (eds.) Efecto de los cambios globales sobre la biodiversidad. CYTED - Programa Iberoamericano de Ciencia y Tecnología para el Desarrollo.

Canavero, A., A. Brazeiro, A. Camargo, I. da Rosa, R. Maneyro, and D. Núñez. 2010a. Amphibian diversity of Uruguay: Background knowledge, inventory completeness and sampling coverage. Boletín de la Sociedad Zoológica de Uruguay 19:2-19.

Canavero, A., S. Carreira, J. Langone, F. Achaval, C. Borteiro, A. Camargo, I. Da Rosa, A. Estrades, A. Fallabrino, F. Kolenc, M. M. López-Mendilaharsu, R. Maneyro, M. Meneghel, D. Núñez, C. M. Prigioni, and L. Ziegler. 2010b. Conservation status assessment of the amphibians and reptiles of Uruguay. Iheringia Sér zool 100(1):5-12.

Carey, C., and M. Alexander. 2003. Climate change and amphibian declines: is there a link? Divers Distrib 9(2):111121.

Carreira, S., and R. Maneyro. 2015. Lista Roja de los Anfibios y Reptiles del Uruguay. Una evaluación del estado de conservación de la herpetofauna de Uruguay sobre la base de los criterios de la Unión Internacional para la Conservación de la Naturaleza. DINAMA. Montevideo, Uruguay.

Caruso, N. M., M. W. Sears, D. C. Adams, and K. R. Lips. 2014. Widespread rapid reductions in body size of adult salamanders in response to climate change. Glob Chang Biol 20:1751-1759.

Chen, C., J. K. Hill, R. Ohlemüller, D. B. Roy, and C. D. Thomas. 2011. Rapid range shift of species associated with high levels of climate warming. Science 333:1024-1026.

Colwell, R., G. Brehm, C. Cardelús, A. Gilman, and J. Longino. 2008. Global warming, elevational range shifts, and lowland biotic attrition in the wet tropics. Science 322:258-261.

D’Amen, M., P. Bombi, P. B. Pearman, D. R. Schmatz, N. E. Zimmermann, and M. Bologna. 2011. Will climate change reduce the efficacy of protected areas for amphibian conservation in Italy? Biol Cons 144:989-997.

Deguise, I., and J. Kerr. 2005. Protected areas and prospects for endangered species conservation in Canada. Conserv Biol 20(1):48-55.

DINAMA. 2005. Análisis de la estadística climática y desarrollo y evaluación de escenarios climáticos e hidrológicos de las principales cuencas hidrográficas el Uruguay y de su Zona Costera (Río Uruguay, Río Negro, Laguna Merín, Río de la Plata y Océano Atlántico). Informe Técnico, Unidad de Cambio Climático. Montevideo, Uruguay. Pp. 84.

Elbers, J. 2011. Las áreas protegidas de América Latina: Situación actual y perspectivas para el futuro. UICN. Quito, Ecuador. Pp. 227.

Elith, J., S. J. Phillips, T. Hastie, M. Dudik, Y. E. Chee, and C. J. Yates. 2011. A statistical explanation of MaxEnt for ecologists. Divers Distrib 17:43-57.

Elith, J., C. Graham, R. Anderson, M. Dudik, S. Ferrier, et al. 2006. Novel methods improve prediction of species' distributions from occurrence data. Ecography 29:129-151.

Franklin, J. 2010. Mapping species distributions: spatial inference and prediction. Cambridge University Press. Cambridge, UK. Pp. 320.

MGAP-FAO. 2013. Clima de cambios: Nuevos desafíos de adaptación en Uruguay. Compilado. Resultado del proyecto fao/ tcp/uru 3302. Montevideo. Disponible libremente en www.fao.org/3/a-au192s.pdf.

Gaston, K., S. F. Jackson, L. Cantú-Salazar, and G. Cruz-Piñón. 2008. The ecological performance of protected areas. Annu Rev Ecol Evol Sys 39:93-113.

Gaston, K. 1994. Rarity. Chapman, and Hall. London, United Kingdom.

Gibbs, J. P., and A. Breisch. 2001. Climate warming and calling phenology of frogs near Ithaca, New York, 1900-1999. Conserv Biol 15:1175-1178.

Gomes-Cortes, L. 2009. Modelagem de distribuição de espécies como ferramenta para a conservação de espécies raras de anfíbios do Cerrado. Tesis de Maestrado, Universidade Federal de Goias, Brasil. Pp. 155.

Halpern, B., S. Walbridge, K. Selkoe, C. Kappel, F. Micheli, et al. 2008. A global map of human impact on marine ecosystems. Science 319:948-952.

Haretche, F., P. Mai, and A. Brazeiro. 2012. Woody flora of Uruguay: inventory and implication within the Pampean region. Acta Bot Bras 26:537-552.

Hernández, P., C. H. Graham, L. Master, and D. Albert. The effect of sample size and species characteristics on performance of different species distribution modeling methods. 2006. Ecography 29:773-785.

Hickling, R., D. Roy, J. Hill, R. Fox, and C. Thomas. 2006. The distributions of a wide range of taxonomic groups are 
expanding polewards. Glob Chang Biol 12:450-455.

Hijmans, R., S. Cameron, J. Parra, P. Jones, and A. Jarvis. 2005. Very high resolution interpolated climate surfaces for global land areas. Int J Climatol 25:1965-1978.

IPCC. 2001. Cambio Climático 2001: Informe de síntesis. Resumen para Responsables de Políticas. IPCC. London, UK.

Kane, R. 2002. Precipitation anomalies in southern America associated with a finer classification of El Niño and La Niña events. Int J Climatol 22:357-373.

Kolenc, F., C. Borteiro, E. M. González, D. A. Barrasso, and C. Prigioni. 2012. Recent findings of the declining frog Pleurodema bibroni Tschudi, 1838 (Anura: Leiuperidae) in Uruguay. Herpetol Notes 5:181-183.

Kolenc, F., C. Borteiro, D. Baldo, D. P. Ferraro, and C. Prigioni. 2009. The tadpoles and advertisement calls of Pleurodema bibroni Tschudi and Pleurodema kriegi (Müller), with notes on their geographic distribution and conservation status (Amphibia, Anura, Leiuperidae). Zootaxa 1969:1-35.

Kottek, M., J. Grieser, C. Beck, B. Rudolf, and F. Rubel. 2006. World Map of the Köppen-Geiger climate classification updated. Meteorol Z 15:259-263.

Langone, J. A., R. Maneyro, and D. Arrieta. 2004. Present knowledge of the status of amphibian conservation in Uruguay. Pp. 82-87 en J. W. Wilkinson (ed.). Collected DAPTF Working Group Reports: Ten years on. DAPTF. Milton Keynes, UK.

Lawler, J. J., S. L. Shafer, D. White, P. Kareiva, E. Maurer, A. R. Blaustein, and P. J. Bartlein. 2009. Projected climateinduced faunal change in the Western Hemisphere. Ecology 90:588-597.

Lobo, J., A. Jiménez-Valverde, and J. Hortal. 2010. The uncertain nature of absences and their importance in species distribution modelling. Ecography 33:103-114.

Loiselle, B. A., C. A. Howell, C. H. Graham, J. M. Goerck, T. Brooks, K. G. Smith, and P. H. Williams. 2003. Avoiding pitfalls of using species-distribution models in conservation planning. Cons Biol 17:1-10.

Lovejoy, T., and L. Hannah. 2005. Climate change and biodiversity. Yale University Press. New Haven, USA. Pp. 418

MacArthur, R. H. 1972. Geographical ecology. Harper and Row. New York, USA. Pp. 269.

Magrin, G., C. Gay García, D. Cruz Choque, J. C. Giménez, A. R. Moreno, G. J. Nagy, C. Nobre, and A. Villamizar. 2007. Latin America. Pp. 581-615 in M. O. Parry, J. Canziani, P. Palutikof, P. van der Linden, and C. Hanson (eds.). Climate Change 2007: Impacts, adaptation and vulnerability. Contribution of working group II to the Fourth assessment report of the Intergovernmental Panel on Climate Change. Cambridge University Press. Cambridge, United Kingdom.

Maneyro, R., and A. Kwet. 2008. Amphibians in the border region between Uruguay and Brazil: Updated species list with comments on taxonomy and natural history (Part I: Bufonidae). Stuttg Beitr Naturkd 1:95-121.

Maneyro, R., and J. A. Langone. 2001. Caracterización de los anfibios de Uruguay. Cuad Herpetol 15:107-118.

Marengo, J., M. Rusticucci, O. Penalba, and M. Renom. 2010. An intercomparison of observed and simulated extreme rainfall and temperature events during the last half of the twentieth century, part 2: historical trends. Clim Chang 98:509-529.

Margules, C., and R. Pressey. 2000. Systematic conservation planning. Nature 405:243-253.

Mason, S. C., G. Palmer, R. Fox, S. Gillings, J. K. Hill, C. D. Thomas, and T. H. Oliver. 2015. Geographical range margins of many taxonomic groups continue to shift polewards. Biol J Linnean Soc 115:586-597.

McCallum, M. 2007. Amphibian decline or extinction? Current declines dwarf background extinction rate. J Herpetol 41:483-491.

Mendonça do Prado, V., F. Rodrigues da Silva, N. Nagatani-Dias, J. Rodrigues-Pires, and D. de Cerqueira-Rossa-Feres. 2009. Anura, Estação Ecológica de Jataí, São Paulo state, southeastern Brazil. Check List 5:495-502.

Mitchell, J. F. B., T. C. John, M. Eagles, W. J. Ingram, and R. A. Davis. 1999. Towards the development of climate change scenarios. Climatic Change 41:547-581.

Myers, N., R. Mittermeier, C. Mittermeier, G. da Fonseca, and J. Kent. 2000. Biodiversity hotspots for conservation priorities. Nature 403:853-858.

Natale, G., and R. Maneyro. 2008. Amphibia, Anura, Leiuperidae, Pleurodema bibroni: Rediscovery. Check List 4:4749.

Núñez, D., R. Maneyro, J. Langone, and R. de Sá. 2004. Distribución geográfica de la fauna de anfibios del Uruguay. Smithsoninan Herpetological Inforamtion Service 134:1-34.

Parmesan, C. 2006. Ecological and evolutionary responses to recent climate change. Annu Rev Ecol Evol Syst 37: 637-669.

Parmesan, C., and G. Yohe. 2003. A globally coherent fingerprint of climate change impacts across natural systems. Nature 421:37-42.

Pearson, R., C. Raxworthy, M. Nakamura, and A. Peterson. 2007. Predicting species distributions from small numbers of occurrence records: a test case using cryptic geckos in Madagascar. J Biogeogr 34:102-117.

Peterson, T., V. Sánchez-Cordero, J. Soberón, J. Bartley, R. Buddemeier, and A. Sanchez-Navarro. 2001. Effects of global climate change on geographic distributions of Mexican Cracidae. Ecol Model 144:21-30.

Phillips, S., M. Dudik, and R. Schapire. 2004. A maximum entropy approach to species distribution modeling. Pp. 655-662 in R. Greiner and D. Schuurmans (eds.). Proceedings of the 21st International Machine Learning Conference. ACM Press. New York, USA. 
Phillips, S., R. Anderson, and R. Schapire. 2006. Maximum entropy modeling of species geographic distributions. Ecol Model 190:231-259.

Phillips, S., and M. Dudik. 2008. Modeling of species distributions with Maxent: new extensions and a comprehensive evaluation. Ecography 31:161-175.

Pineda, E., and J. M. Lobo. 2009. Assessing the accuracy of species distribution models to predict amphibian species richness patterns. J Anim Ecol 78:182-190.

Pounds, J., M. Fogden, and J. Campbell. 1999. Biological response to climate change on a tropical mountain. Nature 398:611-615.

Pounds, J., M. Bustamante, L. Coloma, J. Consuegra, M. Fogden, et al. 2006. Widespread amphibian extinctions from epidemic disease driven by global warming. Nature 439:161-167.

Reading, C. 2007. Linking global warming to amphibian declines through its effects on female body condition and survivorship. Oecologia 151:125-131.

Rosenstock, N., C. Toranza, and A. Brazeiro. 2015. Climate and land-use changes effects on the distribution of a regional endemism: Melanophryniscus sanmartini (Amphibia, Bufonidae). Iheringia Série Zoologia 105:209-216.

Rosset, S. 2008. New Species of Odontophrynus Reinhardt and Lütken 1862 (Anura:Neobatrachia) from Brazil and Uruguay. J Herpetol 42:134-144.

Rusticucci, M., and M. Renom. 2008. Variability and trends in indices of quality-controlled daily temperature extremes in Uruguay. Int J Climatol 28:1083-1095.

Scott, J. M., and J. Schipper. 2006. Gap analysis: a spatial tool for conservation planning. Pp. 518-519 en M. J. Groom, G. K. Meffe and C. R. Carrol (eds.). Principles of conservation biology. Vol. 1. 3a ed. Sinauer Associates, Inc. Sunderland (MA), USA.

Sepúlveda, C., A. Moreira, and P. Villarroel. 1997. Conservación biológica fuera de las áreas silvestres protegidas. Ambient Desarro 13:48-58.

Shmida, A., and M. V. Wilson. 1985. Biological determinants of species diversity. J Biogeogr 12:1-20.

Silvano, D., E. Lavilla, P. García, and J. Langone. 2004. Pleurodema bibroni. The IUCN Red List of Threatened Species. Version 2014.3. www.iucnredlist.org. Visitado: 21 January 2015.

Stuart, S., J. Chanson, N. Cox, B. Young, A. Rodrigues, D. Fischman, and R. Waller. 2004. Status and trends of amphibian declines and extinctions worldwide. Science 306:1783-1786.

Todd, B., T. Luhring, B. Rothermel, and J. Gibbons. 2009. Effects of forest removal on amphibian migrations: implications for habitat and landscape connectivity. J Appl Ecol 46:554-561.

Toranza, C., and R. Maneyro. 2013. Potential effects of climate change on the distribution of an endangered species: Melanophryniscus montevidensis (Anura: Bufonidae). Phyllomedusa 12(2):11-20.

Toranza C., B. Brazeiro, and R. Maneyro. 2012. Efectosdel cambio climático sobre la biodiversidad: El caso de los anfibios de Uruguay. Pp. 22-37 en V. Picasso, L. Astigarraga, G. Cruz y R. Terra (eds.). Cambio y variabilidad climática: Respuestas interdisciplinarias. Espacio Interdisciplinario. Montevideo, Uruguay.

UICN. 2014. The IUCN Red List of Threatened Species. Version 2014.3. www.iucnredlist.org. Visitada: 17 de noviembre de 2014.

Villalobos, F., R. Dobrovolski, D. Provete, and S. Gouveia. 2013. Is rich and rare the common share? Describing biodiversity patterns to inform conservation practices for South American anurans. PLoS ONE 8(2):e56073.

Zank, C., F. G. Becker, M. Abadie, D. Baldo, R. Maneyro, and M. B. Martins. 2014. Climate change and the distribution of Neotropical red-bellied toads (Melanophryniscus, Anura, Amphibia): How to prioritize species and populations? PLoSONE 9(4):1-11.

Zank, C., P. Colombo, G. Adams, D. Borba, and M. Borges-Martins. 2013. New country record and geographic distribution map for the San Martin red-bellied toad Melanophryniscus sanmartini Klappenbach, 1968 (Amphibia, Bufonidae). Herpetol Notes 6:555-557 